\title{
Initiator tRNA genes template the 3' CCA end at high frequencies in bacteria
}

David H. Ardell ${ }^{1,2^{*}}$ and Ya-Ming Hou ${ }^{3}$

\begin{abstract}
Background: While the CCA sequence at the mature $3^{\prime}$ end of tRNAs is conserved and critical for translational function, a genetic template for this sequence is not always contained in tRNA genes. In eukaryotes and Archaea, the CCA ends of tRNAs are synthesized post-transcriptionally by CCA-adding enzymes. In Bacteria, tRNA genes template CCA sporadically.

Results: In order to understand the variation in how prokaryotic tRNA genes template CCA, we re-annotated tRNA genes in tRNAdb-CE database version 0.8. Among 132,129 prokaryotic tRNA genes, initiator tRNA genes template CCA at the highest average frequency (74.1\%) over all functional classes except selenocysteine and pyrrolysine tRNA genes ( $88.1 \%$ and $100 \%$ respectively). Across bacterial phyla and a wide range of genome sizes, many lineages exist in which predominantly initiator tRNA genes template CCA. Convergent and parallel retention of CCA templating in initiator tRNA genes evolved in independent histories of reductive genome evolution in Bacteria. Also, in a majority of cyanobacterial and actinobacterial genera, predominantly initiator tRNA genes template CCA. We also found that a surprising fraction of archaeal tRNA genes template CCA.

Conclusions: We suggest that cotranscriptional synthesis of initiator tRNA CCA 3 ' ends can complement inefficient processing of initiator tRNA precursors, "bootstrap" rapid initiation of protein synthesis from a non-growing state, or contribute to an increase in cellular growth rates by reducing overheads of mass and energy to maintain nonfunctional tRNA precursor pools. More generally, CCA templating in structurally non-conforming tRNA genes can afford cells robustness and greater plasticity to respond rapidly to environmental changes and stimuli.
\end{abstract}

Keywords: Initiator tRNA, Bacteria, CCA

\section{Background}

All active tRNA molecules must contain a CCA sequence at the 3 '-end as the site for amino acid attachment and for interaction with the ribosome during protein synthesis [1-3]. While essential for tRNA activities, the CCA sequence is generally not contained in tRNA genes but is added post-transcriptionally. Exceptions are found in Bacteria, where some tRNA genes contain a template of the CCA sequence for direct synthesis at the time of transcription. However, CCAtemplating is not necessarily conserved among tRNA genes with different functional identities or among

\footnotetext{
* Correspondence: dardell@ucmerced.edu

'Program in Quantitative and Systems Biology, University of California, 5200 North Lake RoadCA 95343 Merced, USA

${ }^{2}$ Molecular and Cell Biology Unit, School of Natural Sciences, University of California, 5200 North Lake Road, Merced, CA 95343, USA

Full list of author information is available at the end of the article
}

bacterial species across different phyla. To explore whether there is potential selective pressure for tRNA genes to template CCA in Bacteria, we undertook a reannotation of publicly available tRNA gene data.

One source of error in the annotation of tRNA genes concerns the functional classification of genes for tRNAs with CAU anticodons. These include genes for initiator tRNA $^{\text {fMet }}$, elongator tRNA ${ }^{\text {Met }}$ and elongator tRNA ${ }_{\text {CAU }}^{\text {Ile }}$ isoacceptors that read AUA codons in Bacteria and Archaea. In this last case of elongator tRNA $A_{C A U}^{\text {Ile }}$, the cytidines at the wobble anticodon position 34 (of the Sprinzl coordinate system for tRNA structure [4]) in transcribed CAU anticodons (C34) are post-transcriptionally modified to lysidine in most Bacteria $[5,6]$, changing the decoding specificity of these tRNAs from AUG to AUA codons. Additionally, this L34 lysidine modification changes the amino acid charging specificity of these tRNAs from methionine to isoleucine by serving as a 
recognition determinant for aminoacylation of tRNA ${ }^{\text {Ile }}$ by the cognate isoleucyl-tRNA synthetase [7]. Archaea use a similar system to decode AUA to isoleucine with a different kind of modification at the same anticodon position [8]. However, currently available tRNA genefinders annotate all three classes as elongator tRNA ${ }^{\text {Met }}$ genes [9]. The TFAM tRNA functional classifier, which uses profile-based models of whole tRNA sequences [10, 11], can differentiate all three tRNA functional classes with generally high specificity and sensitivity (uniformly greater than $98 \%$ depending on data and models used)

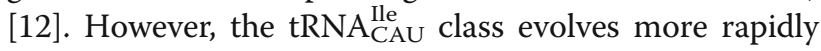
than other classes, so that even though the TFAM 1.4 proteobacterial-specific model generalizes well to some other bacterial phyla, this model does not generalize well to all [13]. An alternative TFAM model [14], for just genes with tRNAs with CAU anticodons, is based on a custom annotation of such genes in a wide sampling of bacterial taxa [12]. Although this alternative model is imperfect in its sensitivity and specificity [12], as discussed further below, its performance is satisfactory and suitable for the present study.

Here we apply this alternative "Silva TFAM model" to improve the functional annotation of tRNA genes with CAU anticodons in the high quality public database tRNAdb-CE [15, 16]. In our analysis, we found that genes for the initiator class of tRNAs across the bacterial domain consistently template CCA with significantly higher frequencies than elongator tRNA genes. This CCA-templating can provide unique advantages to initiator tRNA for rapid maturation, aminoacylation, and initiation of protein synthesis.

\section{Results}

Functional reannotation of bacterial genes in tRNAdb-CE v.8 The tRNAdb-CE v0.8 database contains 132,129 total prokaryotic gene records, of which 129,989 gene records are bacterial. Among the bacterial sequences, 9,917 contain a template for anticodon with sequence CAU. For a substantial fraction of its records, the tRNAdb-CE v0.8 database uses TFAM 1.4 for functional classification of bacterial tRNA genes (as described in http://trna.ie.niigata-u.ac.jp/ trnadb/method.html). However, the Proteobacterial model for the tRNA ${ }_{\text {CAU }}^{\text {lle }}$ elongator class that comes with TFAM 1.4 does not generalize well to all bacterial phyla [13]. Therefore, we reannotated the 9,917 bacterial genes for tRNAs with CAU anticodons in tRNAdb-CE v0.8 using the more general Silva TFAM model derived from the analysis of Silva et al. [12]. This model is also provided as supplementary data in the present work. By applying the Silva TFAM model to 9,917 bacterial CAU-anticodon containing tRNA genes, we revised the functional annotations of 62 of 3,918 previously annotated genes $(\approx 1.58 \%)$ and provided new annotations for 5,999 additional genes that were previously unspecified ( $\approx 60.4 \%)$. Reclassification frequencies are presented in Table 1 , showing that no previously classified genes were reannotated as initiator tRNA ${ }^{\text {MMet }^{-}}$ genes and that our reannotation efforts resulted in approximately equal frequencies of the two elongator tRNA gene classes. These reannotated data are also provided in Additional files 1 and 2 .

\section{Structural reannotation of bacterial genes in tRNAdb-CE v.8} A well-designed feature of the tRNAdb-CE v0.8 data model lies in that its gene records contain not only annotated gene sequences but also ten bases of genomic context both up- and downstream. Inspection of tRNAdb-CE v0.8 data revealed multiple genes with an annotated 3 '-end sequence other than CCA, followed by 3 '-trailer sequences that begin with the sequence CCA. To confidently assess whether these genes might template a 3 '-CCA-end for their gene products, we assigned Sprinzl coordinates [4] to these bases for each gene sequence. These coordinates were not provided in tRNAdb-CE v0.8. We did this by implementing a dynamic programming algorithm to optimize base-pairing of the acceptor 3 '-end region against the databaseannotated 5 '-end. Our dynamic programming algorithm with parameters given in methods (which were chosen somewhat arbitrarily and not optimized) almost always annotated acceptor stems identically to those given in the database. Also, we never used our algorithm to reannotate acceptor stems, only to confidently and consistently assign Sprinzl coordinates to the 3 '-end region of each gene. Using this technique, we annotated an additional 2,866 bacterial tRNA genes out of 129,989 (or $2.2 \%$ ) records as containing the CCA template at the 3 '-end in the sequence framework of Sprinzl coordinates 74-76.

To clarify why we could identify an additional 2,866 tRNA genes in tRNAdb-CE v0.8 that template CCA, we ran tRNA gene-finding programs on the database records, suspecting that perhaps user error with tRNA gene-finders may have caused these misannotations. We used ARAGORN v1.0 [17] and tRNAscan-SE v.1.23 [18] in default eukaryotic tRNA gene-finding mode, and

Table 1 Reannotation of bacterial tRNAs with CAU anticodons in tRNAdb-CE v.8 using a custom model based on the analysis of Silva et al. [12]. "klle" means Isoleucine tRNA elongators with CAU anticodon. "???" means that no specific annotation was provided in tRNAdb-CE v.8

\begin{tabular}{llllll}
\hline & & Met & Ini & klle & Sum \\
\hline Annotations & Met & 1055 & 0 & 51 & 1106 \\
In tRNAdb-CE & Ini & 0 & 1824 & 0 & 1824 \\
v.8 & klle & 11 & 0 & 977 & 988 \\
& ??? & 1696 & 2614 & 1689 & 5999 \\
& Sum & 2762 & 4438 & 2717 & 9917 \\
\hline
\end{tabular}


tRNAscan-SE v.1.23 in Bacterial mode (with the -B option). We found that tRNAscan-SE v.1.23, when run in its default eukaryotic gene-finding mode, never annotates nucleotides at positions 74-76 irrespective of their sequence. An exception to this rule was with selenocysteine tRNA genes, for which tRNAscan-SE in eukaryotic mode does annotate positions 74-76 if they contain the CCA sequence. From these observations we conclude that a likely cause of misannotations in tRNAdb-CE is user error in genome annotation pipelines. This is particularly notable when users of tRNAscan-SE apply its default eukaryotic gene-finding mode on prokaryotic genomes. Such errors may then be incessantly propagated in public and private databases.

\section{Frequencies of CCA-templating in bacterial tRNA genes}

With our reannotated tRNAdb-CE data in hand, we calculated frequencies of CCA-templating in tRNA genes across different tRNA functional classes and taxonomic groupings as defined by NCBI Taxonomy [19]. Figure 1 shows our data summarized by prokaryotic genus. Prokaryotic clades exhibit all four possible patterns: 1) most or all tRNAs genes template CCA, 2) few or no tRNA genes template $\mathrm{CCA}, 3)$ primarily initiator genes template $\mathrm{CCA}$, or - most rarely -4 ) primarily elongator tRNA genes template CCA.

The five best-sampled phyla in our dataset, as defined by number of distinct genera with at least one genome sequenced, are Proteobacteria, Bacillus/Clostridium, Actinobacteria, Bacteroidetes/Clorobi, and Cyanobacteria. These five phyla exhibit three of four patterns described above in a strikingly consistent pattern by phylum. Practically all tRNA genes template CCA in Proteobacteria and Bacillus/Clostridium, except in certain reduced genomes, most of which template CCA only in initiator tRNA genes, or in no tRNA genes at all. In Cyanobacteria and Actinobacteria, on the other hand, primarily only the initiator tRNA genes template CCA, with certain exceptions. For example, a clade of Actinobacteria with relatively small genomes exists in which both initiator and elongator tRNA genes template CCA at high frequencies. In the Bacteroidetes/Chlorobi group, most tRNA genes do not template CCA, except for one lineage, the Solitalea, in which only initiator tRNA genes template CCA. In all five of the most-sampled phyla, there exist both small and moderately-sized genomes in which only initiator tRNA genes template CCA, or no tRNA genes at all template CCA. Certain Myxococcales, among the Deltaproteobacteria, are exceptional in having among the largest genomes that we observed and yet no tRNA genes or only initiator tRNA genes template CCA.

Less-sampled phyla are also quite heterogeneous in our dataset. In the Thermotogae, Deinococcus/Thermus and Tenericutes, all tRNA genes template CCA.
Spirochaetae do not template CCA in any genes, while in Deferribacteres, only initiator tRNA genes template CCA. In the most rare pattern we observed, in only a few archaeal or bacterial genera, primarily elongator tRNA genes and not initiator tRNA genes template CCA.

In order to better visualize these data down to individual genomes and separating different elongator classes, we created an interactive javascript-based taxonomic navigator for our results visualized with heatmaps in any ordinary web browser. The full interactive data navigator is available as Additional files $1,2,3,4,5,6,7,8,9,10$, 11,12 , and 13 to this work. A static view on these data is also provided as a searchable PDF in Additional files $1,2,3,4,5,6,7,8,9,10,11,12,13$ and 14 . Figure 2 presents a snapshot from this browser with some notable detailed results for Bacterial tRNA genes. The figure shows columns of frequency data, corresponding to functional classes of tRNA genes, sorted left to right by decreasing average frequency at which tRNA genes template CCA over all prokaryotic genomic sequences in our sample. This analysis reveals that, in Bacteria and Archaea, initiator tRNA genes (labeled as Ini) template CCA at the highest frequency (74.1\%), versus $66.2 \%$ for elongator tRNA genes generally. In Bacteria, initiator tRNA genes template CCA at a frequency of $74.7 \%$, second only to selenocysteinyl tRNA genes $\left(\mathrm{tRNA}^{\mathrm{Sec}}, \mathrm{Sec}=\right.$ selenocysteine), with a frequency of $89.2 \%$. We also found that genes for tRNA ${ }^{\text {Asp }}$ and tRNA ${ }^{\text {Asn }}$ template CCA at the highest frequencies among all canonical elongator tRNA genes. Below we describe some of the notable results shown in Fig. 2.

\section{Cyanobacteria}

Among bacterial phyla we observed, Cyanobacteria have the most striking and consistent pattern in which specifically initiator and not elongator tRNA genes template CCA. The overall frequencies are $64.1 \%$ for initiator tRNA genes versus $25.7 \%$ for the next highest gene class, which are elongator tRNA ${ }^{\text {Tyr }}$ genes. But different cyanobacterial lineages exhibit considerable variation in this trait. For example, among Prochlorales and Nostocales genomes - comprising both the smallest and largest average genome sizes, respectively - the frequencies at which initiator tRNA genes template CCA are 91.7\% and $88.9 \%$, while elongator tRNA genes template CCA at only $3.4 \%$ and $8.2 \%$ respectively. In 11 out of 12 of Prochlorococcus genomes and 9 out of 13 Synechococcus genomes, only initiator tRNA genes template CCA. Initiator tRNA genes template CCA at very different frequencies in sister orders Oscillatoriales and Chroococcales within subclass Oscillatoriophycideae: 87.5 and $43.8 \%$ respectively.

The Cyanobacteria are also unusual in that different strains and groups feature specific elongator tRNA gene 


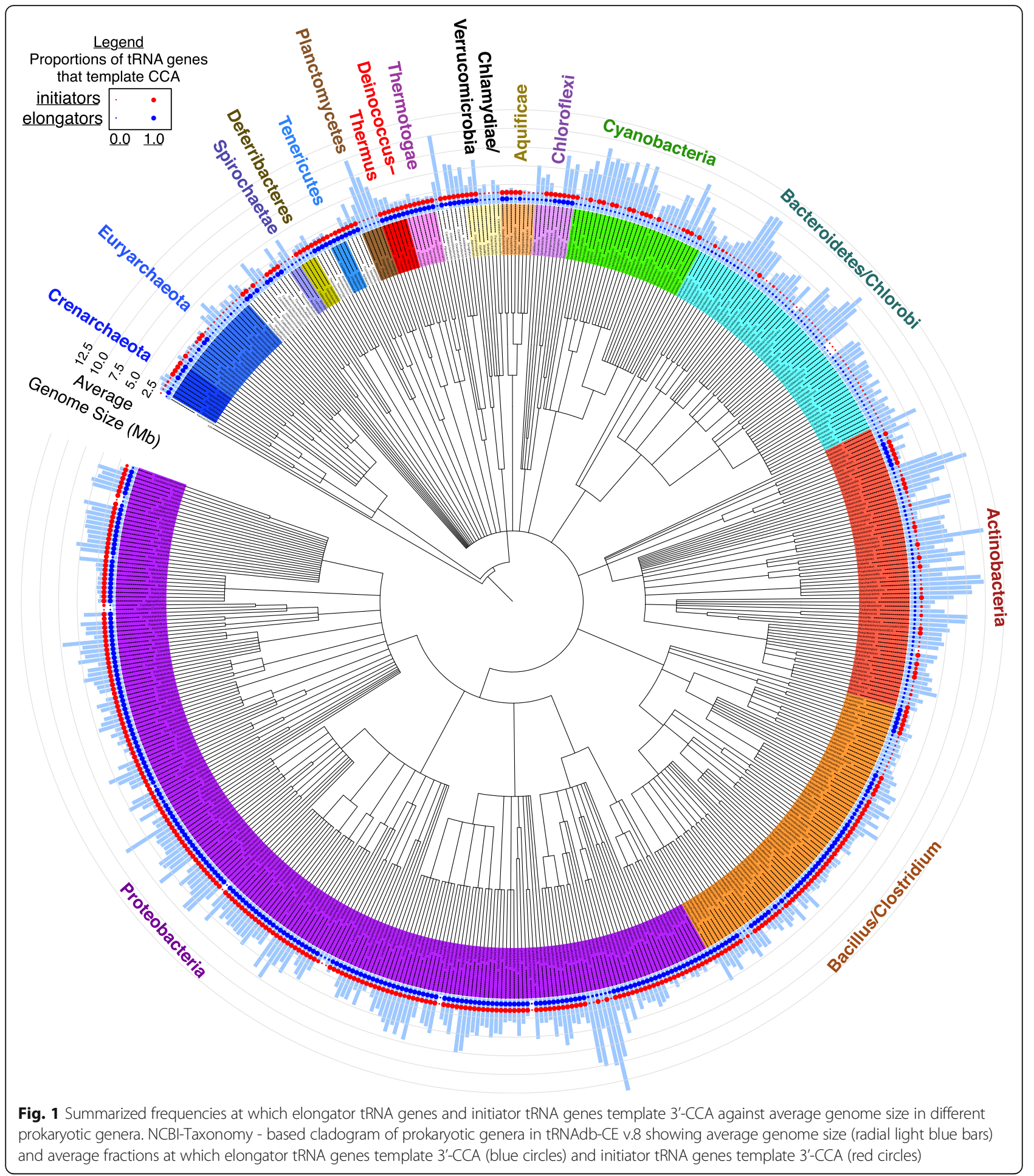

classes that also template CCA at intermediate frequencies (above 10\%) while other elongator classes template CCA at lower frequencies (below 10\%). Usually, if in any one genome the initiator tRNA gene or genes template CCA, at least one elongator tRNA gene class will also template CCA at an intermediate frequency. The elongator gene class that templates CCA most consistently across the phylum is the tRNA ${ }^{\text {Tyr }}$ gene class. In Nostocales, $\mathrm{tRNA}^{\mathrm{Tyr}}$ genes template CCA at a frequency of $(41.7 \%)$, while tRNA ${ }^{\text {Asn }}$ and tRNA ${ }^{\text {Gln }}$ elongator genes also template CCA at a high relative frequency (36.8\% and $23.7 \%$ ). 


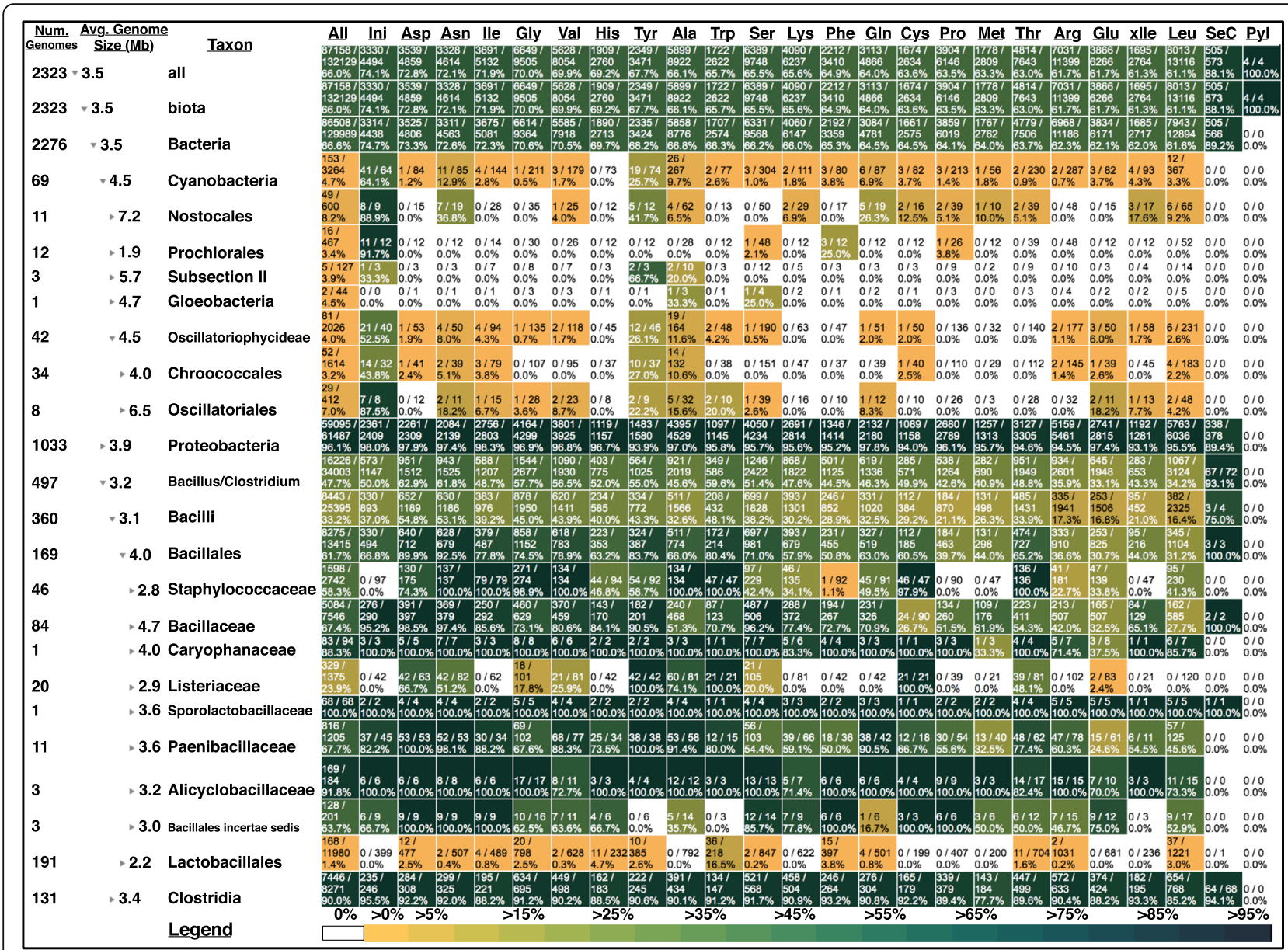

Fig. 2 Summarized genome size and CCA frequency data in Bacterial clades broken out by tRNA functional class. Except for columns labelled "All," "SeC," and "Pyl," all columns of frequency data are sorted in decreasing order from left to right by the frequency at which tRNA gene classes template CCA over all prokaryotic genomes that we sampled. Clades are defined as in NCBI Taxonomy. Column labels correspond to IUPAC three-letter amino acid charging identity except for "Ini" (initiators) and "xlle" (AUA-codon-reading isoleucine isoacceptors). "All" summarizes frequency data over all tRNA classes

\section{Proteobacteria}

All proteobacterial tRNA genes generally template CCA at consistently high frequencies: $96.1 \%$ overall (Fig. 2). Yet proteobacterial initiator tRNA genes template CCA at $98.0 \%$, significantly higher than proteobacterial elongators $\left(G=29.039\right.$, d.f. $=1, p<10^{-6}$ by a G-test of independence computed in R 3.3.1 using function "G.test" in package "RVAideMemoire" version 0.9-60). Closer examination of the proteobacterial variation (Additional file $1,2,3,4,5,6,7,8,9,10,11,12,13$ and 14) reveals that while many free-living proteobacteria template CCA at high frequencies, endosymbiotic $\gamma$-proteobacteria and $\alpha$-proteobacteria with reduced genomes show similar patterns to those described above for cyanobacteria with reduced genomes. In these cases, initiator tRNA genes appear to be the only class to consistently template CCA, while several elongator classes also template CCA. For example, in most Buchnera aphidicola genomes, about eight or nine additional elongator tRNA classes template CCA at intermediate to high frequencies while other classes do not template CCA, as previously reported [20]. Remarkably, in all Buchnera strain genomes except one, initiator tRNA genes always template CCA. Furthermore, like in the Cyanobacteria, in the smallest of the Buchnera genomes, only initiator tRNA genes template CCA. This same pattern holds in other endosymbiotic $\gamma$-proteobacteria genomes such as Ca. Blochmannia, Wigglesworthia, Glossina, Ca. Baumannia, Ca. Carsonella, Ca. Portiera, as well as $\alpha$-proteobacteria endosymbionts such as Wolbachia. In contrast, among the smallest $\gamma$-proteobacterial genomes like Ca. Hodgkinia, none of the tRNA genes template CCA.

\section{Other bacterial phyla}

Many diverse genera and classes of Bacteria preferentially template CCA in their initiator tRNA genes 
(Additional file 1, 2, 3, 4, 5, 6, 7, 8, 9, 10, 11, 12, 13 and 14). Examples include Geobacillus, Thermoaerobacter, Ruminococcus, Thermomicrobiales, Deferribacter, Thermodesulfobacteria, Mycobacterium, Propionibacterium, Frankia, and Bifidobacterium. As shown in Fig. 2, within the Bacillus/Clostridium phylum, frequency variation in this genomic trait is highly extensive. An unusual pattern is found in the pathogenic Staphylococcaeceae and Listeraceae families, and also the Lactobacillales, which contain both pathogens and non-pathogens, in which initiator tRNA genes never template CCA, even while elongator tRNA genes do template CCA at intermediate frequencies. For example, in Staphylococcaceae about $60 \%$ of elongator tRNA genes template CCA and in Listeraceae about $24 \%$ of elongator tRNA genes template CCA, while in Lactobacillales, $1.4 \%$ of elongator genes template CCA. Yet among the 257 genome representatives of these three families in our dataset, not one initiator tRNA gene templates CCA.

\section{Archaea}

We found no need for structural or functional reannotation of archaeal tRNA genes in tRNAdb-CE v.8. Figure 3 presents a snapshot from our data browser (Additional file 11) with some of our most notable results for archaeal tRNA genes. While there are fairly high frequencies of CCA-templating in archaeal tRNA genes overall, at 30.4\%, we found that initiator tRNA genes in Archaea do not template CCA at any especially high frequency among tRNA genes, which presents a major difference from Bacteria. Other than this, we observed extensive phyletic variation in this trait across Archaea. Crenarchaeota tRNA genes template CCA at a frequency of 50.5\%, while Euryarchaeota tRNA genes template CCA at about half of that frequency. Within Crenarchaeota, tRNA genes in the Sulfolobales template CCA at 3.8\%, but in the Desulforococcales this frequency is $84.8 \%$. All four tRNA ${ }^{\mathrm{Pyl}}$ (Pyl = pyrrolysine) genes template CCA in the Methanomicrobia. Contrary to the generalization that Archaea tRNA genes do not template CCA, there exist lineages in both the Crenarchaeota and Euryarchaeota in which all or nearly all tRNA genes template CCA, for example in the Desulfurococcales, Protoarchaea, and Methanopyri. Although variation exists across tRNA functional classes in a phyletic pattern, no obvious overall pattern emerges.

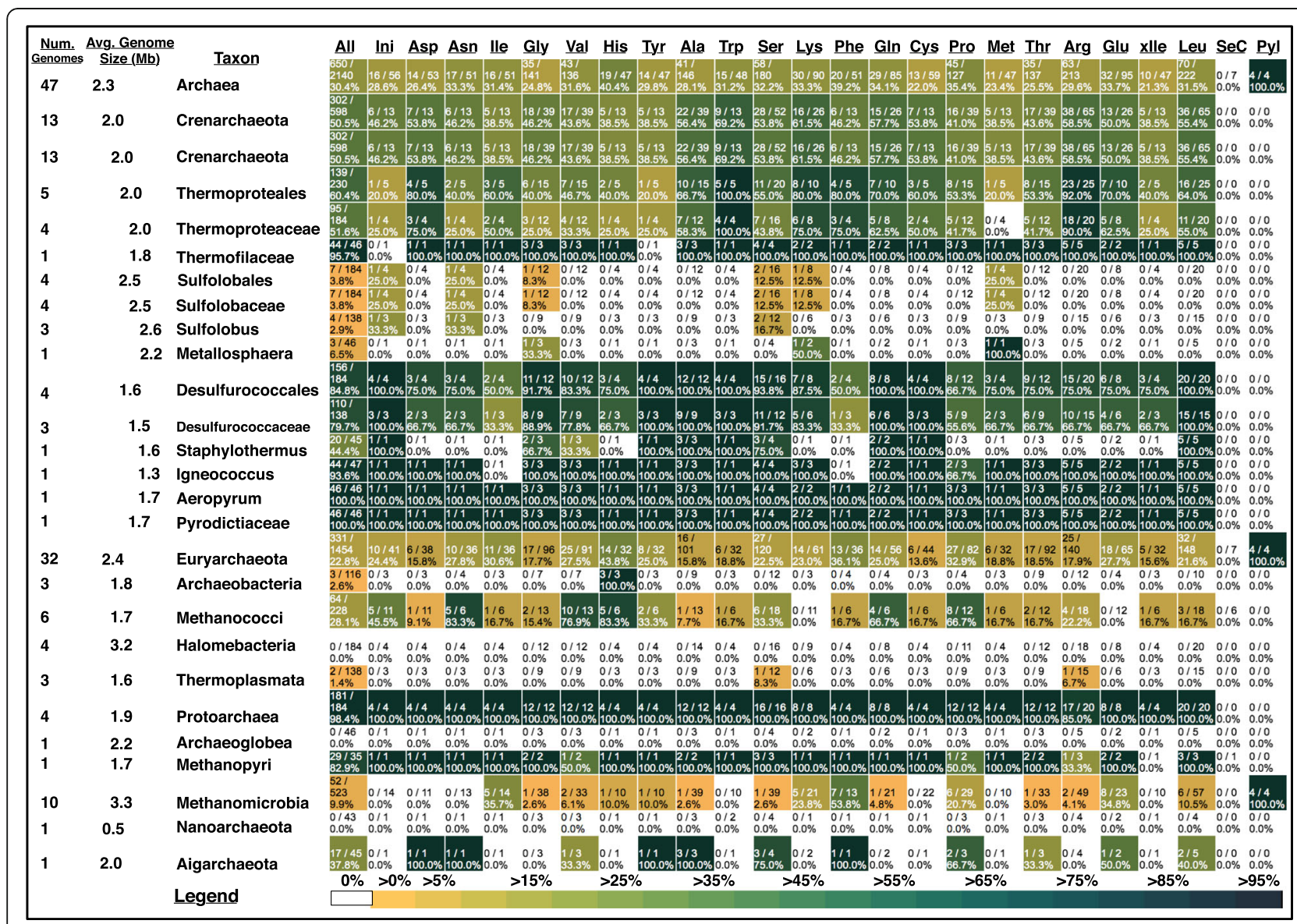

Fig. 3 Summarized genome size and CCA frequency data in Archaeal clades broken out by tRNA functional class. Annotations are the same as in Fig. 2 


\section{Discussion}

With the rapid accumulation of whole-genome sequencing data in recent years, it has become increasingly critical to design strategies that aid in proper genome annotation at large scales. One of the most challenging issues is consistent annotation of tRNA genes. We describe here a strategy that addresses this challenge, particularly with the goal of consistently annotating initiator $v s$. elongator tRNA ${ }^{\text {Met }}$ genes. Using our new strategy, we observed widespread phyletic variation in the frequencies and patterns at which tRNA genes template CCA across functional classes in prokaryotic genomes. Across diverse bacterial and archaeal clades, frequencies range between 0 to $100 \%$. The key finding is that initiator tRNA genes have the greatest class-specific frequency of CCA-templating in Bacteria after tRNA ${ }^{\mathrm{Sec}}$ genes. Furthermore, in diverse bacterial lineages, especially among the reduced genomes of free-living Cyanobacteria and host-associated endosymbiotic Proteobacteria, initiator tRNA genes template CCA at uniquely high frequencies. In Proteobacteria, all tRNA genes template CCA at high frequencies, but initiator tRNA genes have the highest overall frequency, second only to tRNA ${ }^{\text {Sec }}$ genes.

The tRNA gene reannotations that led to our results are meaningful. The most likely source of reannotation errors would be from our reclassification of tRNA gene function (Table 1). Note that no previously annotated initiator tRNA genes were reclassified in our analysis, but rather a substantial fraction of previously unclassified genes were annotated. With respect to these new classifications, detection of initiator tRNA genes by TFAM has high sensitivity and specificity, of less than $1 \%$ false negatives or positives respectively [10, 12]. This is because initiator tRNAs contain features that distinguish them from elongator tRNAs in highly conserved and domain-specific ways [21]. As shown in Tables 2, 3 and 4, our initiator tRNA gene predictions confirm and extend previously described rules and exceptions for bacterial initiator tRNA genes [21], including distinct patterns of mismatching at coordinates 1 and 72 in the acceptor stem (Table 2), distinct nucleotide frequencies at the 11:24 base-pair in the D-stem (Table 3), and unique anticodon-stem statistics at positions $29-31$ and 39-41 (Table 4).

Furthermore, our results are robust to missing data. For example, we were unable to annotate any initiator tRNA genes in one of the Cyanobacterial lineages shown in Fig. 2 - Gloeobacter. In our analysis of the tRNA gene complements of 2,323 prokaryotic genomes in tRNAdb-CE v.8, we were unable to annotate initiator tRNAs genes in 15 genomes $(0.6 \%$; Additional files 1, 2, $3,4,5,6,7,8,9,10,11,12,13$ and 14). We spotchecked several of these aberrant tRNA gene complements by examining their score distributions with TFAM
Table 2 Frequencies of bases at coordinates 1 and 72 in the acceptor stem of reannotated tRNA genes of tRNAdb-CE v.8

\begin{tabular}{|c|c|c|c|c|c|}
\hline & & Met & Ini & klle & Sum \\
\hline \multirow[t]{17}{*}{$1: 72$} & $C A$ & 2 & 3392 & 1 & 3395 \\
\hline & $\mathrm{CU}$ & 0 & 626 & 0 & 626 \\
\hline & $\mathbf{U} \mathbf{U}$ & 1 & 207 & 1 & 209 \\
\hline & C C & 0 & 90 & 2 & 92 \\
\hline & $\underline{U: A}$ & 2 & 63 & 1 & 66 \\
\hline & $A A$ & 0 & 39 & 2 & 41 \\
\hline & U.G & 1 & 9 & 2 & 12 \\
\hline & G A & 0 & 7 & 0 & 7 \\
\hline & U C & 1 & 3 & 3 & 7 \\
\hline & $A C$ & 0 & 1 & 6 & 7 \\
\hline & A G & 0 & 1 & 1 & 2 \\
\hline & G.U & 1371 & 0 & 103 & 1474 \\
\hline & $\underline{\mathrm{G}: \mathrm{C}}$ & 1287 & 0 & 2511 & 3798 \\
\hline & $\underline{\mathrm{C}: \mathrm{G}}$ & 66 & 0 & 77 & 143 \\
\hline & $\underline{A: U}$ & 30 & 0 & 6 & 36 \\
\hline & SC & 1 & 0 & 0 & 1 \\
\hline & G G & 0 & 0 & 1 & 1 \\
\hline Sum & & 2762 & 4438 & 2717 & \\
\hline
\end{tabular}

Watson-Crick matching base-pairs are underlined and mismatches are in bold-face

0.4 to verify that there were no viable candidates for initiator tRNA genes according to our models. All of the tRNA genes in these gene complements that we checked scored inside of the normal background distribution for the initiator tFAM model $[10,12]$. We believe that initiator tRNA genes may simply be missing from the genome annotations that were aggregated in tRNAdb-CE v.8. Importantly, in these genomes, the pattern by which tRNA genes template CCA is phylogenetically consistent with related genomes (Fig. 2 and Additional files 1, 2, 3, $4,5,6,7,8,9,10,11,12,13$ and 14).

Table 3 Frequencies of base-pairs at coordinates 11:24 in the D-stem of reannotated tRNA genes of tRNAdb-CE v.8

\begin{tabular}{clllll}
\hline & & Met & Ini & klle & Sum \\
\hline $11: 24$ & A:U & 0 & 4430 & 0 & 4430 \\
& $\frac{\mathrm{C}: \mathrm{G}}{\mathrm{G}: \mathrm{C}}$ & 2759 & 3 & 2706 & 5468 \\
& - & 0 & 3 & 0 & 3 \\
& -- & 1 & 1 & 0 & 2 \\
& U:A & 1 & 1 & 8 & 10 \\
& U.G & 1 & 0 & 2 & 3 \\
& C A & 0 & 0 & 1 & 1 \\
& & 2762 & 4438 & 2717 & 9917 \\
\hline
\end{tabular}

Watson-Crick matching base-pairs are underlined 
Table 4 Frequency of base-pairing sequences at coordinates 29-31 and 39-41 in the coding stem of reannotated tRNA genes of tRNAdb-CE v.8. All sequences that appear more than 100 times in total are shown; complete data are shown for initiator tRNA genes

\begin{tabular}{llllll}
\hline & & Met & Ini & klle & Sum \\
\hline 29-31,39-41 & GGG,CCC & 2 & 3849 & 1 & 3852 \\
& AGG,CCU & 1 & 522 & 1 & 524 \\
& AGG,UCU & 6 & 34 & 0 & 40 \\
& GGG,UCC & 0 & 18 & 0 & 18 \\
& CGG,CCG & 869 & 0 & 974 & 1843 \\
& UCA,UGA & 405 & 0 & 0 & 405 \\
& GCA,UGC & 369 & 0 & 0 & 369 \\
& GGA,UCC & 335 & 0 & 583 & 918 \\
& CGA,UCG & 213 & 0 & 654 & 867 \\
Sum & GGA,CCC & 114 & 0 & 75 & 189 \\
& & 2314 & 4438 & 2286 & 9038 \\
\hline
\end{tabular}

The initiator tRNA of protein synthesis in Bacteria is known as tRNA ${ }^{\text {fMet }}$ because its charged methionine moiety contains a formyl group attached to the $\alpha$-amino group. By templating CCA in tRNA ${ }^{\text {fMet }}$ genes, Bacteria can directly synthesize tRNA ${ }^{\text {fMet }}$ with the CCA sequence at the 3 '-end. Below we hypothesize five non-mutually exclusive potential advantages for tRNA genes to template CCA.

Our first hypothesis is that certain tRNA classes, particularly initiator tRNAs, may have relatively nonconforming structures that lead to inefficient processing in shared tRNA maturation pathways. For example,

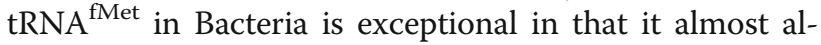
ways contains a mismatch pair at the $1-72$ position of the acceptor end. In our data (Table 2), 98.6\% of our predicted bacterial initiators contain a mismatch at coordinates 1 and 72, and among those, 77.5\% contain the C1-A72 mismatch, which has been specifically shown to promote recognition by initiation factors to initiate protein synthesis in E. coli [22]. Only 66 of 4438 (1.4\%) of predicted initiators contain a Watson-Crick (W-C) or wobble match at these positions, and these all contain the U1-A72 W-C match, which is almost completely absent in any elongator tRNAs (only 3 of 5179 predicted elongators or about $0.05 \%$ ). In contrast, $99.5 \%$ of our predicted elongator tRNAs contain a Watson-Crick (WC) or wobble base pair at the 1-72 position which may be important for them to be discriminated against by initiation factors. Critically, because the C1-A72 mismatch motif of tRNA ${ }^{\text {fMet }}$ is known to compromise the efficiency of processing at the $5^{\prime}$-end [23], we suggest that direct transcription of the CCA sequence can serve as a determinant to help with 5 '-end processing [24, 25]. In contrast, initiator tRNAs in Archaea have Watson-
Crick base-pairs in the 1-72 position [21] so we conjecture that they do not share this "Achilles heel" problem with Bacterial initiator tRNAs, but instead are efficiently processed at the $5^{\prime}$-end without any requirement for a 3 '-end CCA sequence. This is consistent with our observation that initiator tRNA genes in Archaea do not have a particularly high frequency of CCA-templating. In contast, we find that both $\mathrm{tRNA}^{\mathrm{Sec}}$ genes and $\mathrm{tRNA}{ }^{\text {Pyl }}$ genes have high frequencies of CCA-templating in bacteria, consistent with the notion that both $\mathrm{tRNA}^{\mathrm{Sec}}$ and tRNA ${ }^{\text {Pyl }}$ have unusual structures that should compromise 5'-end processing. Specifically, tRNA ${ }^{\text {Sec }}$ is unique in having 8 base-pairs in the acceptor stem, in contrast to 7 base-pairs in all other tRNAs. Similarly, tRNA ${ }^{\text {Pyl }}$ is characterized by the absence of the conserved G18-G19 bases in the Dloop and the absence of the TC sequence in the T loop. All of these unusual features can alter the structure of the acceptor-T stem loop, leading to discrimination by the $5^{\prime}$ end processing enzyme RNase P [26]. By having the CCAtemplate, both $\mathrm{tRNA}^{\mathrm{Sec}}$ and $\mathrm{tRNA}^{\text {Pyl }}$ can use the transcribed CCA sequence to mitigate the reduced efficiency with 5 '-end processing. Additionally, because the CCAadding enzyme (conserved in evolution) recognizes the acceptor-T stem-loop structure to catalyze CCA addition [27], the alteration of the structure by abberant features in tRNA $^{\text {fMet }}$, tRNA ${ }^{\mathrm{Sec}}$, and $\mathrm{tRNA}^{\mathrm{Pyl}}$ may compromise the activity of the CCA enzyme. Thus direct templating of CCA provides a solution to circumvent the problem.

Second, direct templating of the CCA sequence in tRNAs can potentially increase the maximal growth rate of cells. Under conditions of rapid growth, the cotranscriptional synthesis of 3 '-terminal CCA in tRNAs can increase the allocation of cellular resources directly to the synthesis of new proteomic biomass and growth in two ways: first, by reducing steady-state cellular pools of species of nonfunctional tRNA precursors, which reduces the mass and energy overhead of the translational machinery itself, and second, by reducing the steady-state fraction of ribosomes devoted to synthesizing tRNA-affiliated proteins such as CCA-adding enzyme [28, 29].

Third, given that translational initiation is rate-limiting in protein synthesis [30], and therefore a key determinant of maximal growth rate [28, 31, 32], cells selected for a high maximum growth rate may need to efficiently maintain high concentrations of mature initiator tRNA ${ }^{\text {fMet }}$ for rapid growth. The costs of maturation of a tRNA to a growing cell should increase proportionally with the concentration of that tRNA, and initiator tRNA concentration increases more with growth rate in $E$. coli than elongator tRNA concentration [33]. Thus, the fitness impact of templating CCA on initiator tRNAs should be greater than on elongator tRNAs in rapidly growing cells. For example, the record-high growth rates reported among Vibrio species [34] is associated with very high initiator tRNA gene 
copy numbers in Vibrio genomes [10]. Consistent with the above, all initiator tRNA genes in Vibrio template CCA in the present analysis.

Fourth, rapid synthesis of initiator tRNAs through cotranscriptional synthesis of CCA could reduce the lag phase associated with the transition to growth by reducing the waiting time to increase initiator tRNA concentration. Importantly, this "bootstrapping" trait may be important for all cells, including free-living and endosymbiotic Bacteria under reductive genome evolution, and not just for cells capable of rapid growth. Many such cells could have an advantage in the rapid initiation of protein synthesis from a quiescent state in response to environmental change. Indeed, we have shown that each nucleotide addition for post-transcriptional synthesis of CCA requires the CCA enzyme to proofread tRNA integrity $[35,36]$, which likely delays maturation of newly transcribed tRNAs.

Fifth, for elongator tRNA genes, direct templating of CCA can facilitate more rapid synthesis of the corresponding elongator tRNAs to help cells avoid transient depletion of specific ternary complexes and the detrimental consequences that such shortages may have on the accuracy of protein synthesis and proteomic integrity. The supply-demand theory of tRNA charging dynamics [37] predicts wide variability in the sensitivity of charging levels of tRNA species to perturbations, such as amino acid starvation, affecting specific elongator tRNAs for both proteomically abundant and rare amino acids such as Leucine, Tyrosine and Phenylalanine. Stalled ribosomes caused by shortages of specific ternary complexes increase translational misreading at corresponding "hungry" codons $[38,39]$, including frame-shift errors [40], all of which can cause protein misfolding, aggregation, and damage [41].

While many cyanobacteria with reduced genomes are not fast-growing, they may generally be subject to multiple constraints of chronic nutrient limitation and a heavy burden of a large fraction of proteome dedicated to autotrophic functions [42]. When combined, these factors may lead to "proteomic constraints" from small cell sizes, an exacerbation of macromolecular crowding, and increased sensitivity to mistranslation of the most abundant parts of the proteome [42]. We suggest that the relatively high frequency at which $\mathrm{tRNA}^{\mathrm{Tyr}}$ genes template CCA in Cyanobacteria (Fig. 2) is associated with a unique biological sensitivity to depletion of charged tRNA ${ }^{\mathrm{Tyr}}$. Tyrosine residues are critically important for both catalysis and stability of RuBisCo [43], one of the most abundant proteins in Cyanobacteria [44]. It is remarkable that there exists a D-Tyr-tRNA ${ }^{\text {Tyr }}$ deacylase that is conserved and apparently unique to Cyanobacteria [45], which helps maintain the accuracy of tRNA $^{\text {Tyr }}$ charging. Competition experiments that model biologically relevant conditions with Cyanobacterial strains with or without CCA-templating for tRNA ${ }^{\text {Tyr }}$, as well as biochemical assays, could test this hypothesis.

We further suggest that the advantages of avoiding supply shortages and streamlining tRNA biogenesis pathways may extend to other elongator tRNAs that we found to template CCA in an often lineage-specific manner. Selenocysteine and Pyrrolysine tRNAs both have complex biosynthetic/maturation pathways and both template CCA at high frequencies in our analysis. Similarly, biosynthesis of Asn-tRNA ${ }^{\text {Asn }}$ involves two steps, first by synthesizing a mispaired Asp-tRNA ${ }^{\text {Asn }}$, followed by conversion of Asp to Asn [46-48]. Indeed, genes for both tRNA ${ }^{\text {Asp }}$ and tRNA ${ }^{\text {Asn }}$ template CCA at high frequencies. Although the synthesis of Gln-tRNA ${ }^{\text {Gin }}$ also relies on a two-step pathway involving transamidation of Glu on Glu-tRNA ${ }^{\text {Gln }}$ [49], the frequencies for tRNA ${ }^{\text {Glu }}$ and tRNA ${ }^{\text {Gln }}$ are among the lowest we observed in Bacteria overall. Further analysis and experiments will be necessary to fully understand the patterns reported in this paper.

We have not examined trends of CCA-templating in eukaryotic tRNA genes as these data are not currently included in tRNAdb-CE. Although the hypotheses we have described for our results in Bacteria are all potentially applicable to eukaryotes, we anticipate that eukaryotic tRNA genes may exhibit entirely distinct trends in structural variation. In bacteria, our hypotheses could be further investigated computationally through phylogenetically informed comparative analysis of bacterial traits including metagenome data, and through the genomically informed simulation of whole-cell models of gene expression in bacteria.

\section{Conclusions}

Re-annotation of tRNA gene sequences was essential to our discovery that CCA-templating is a major feature of initiator tRNA genes in Bacteria. This shows the importance for genome annotation projects of using tRNA gene-finders with taxonomically correct models. More generally, this work demonstrates the importance of using bioinformatic assets carefully to maximize scientific returns.

\section{Methods}

\section{Data}

Version 8 (October, 2014) of the tRNAdb-CE database [16] was downloaded on November 4, 2014. NCBI Taxonomy data [19] was downloaded on November 13, 2014.

\section{Functional reannotation of CAU-anticodon tRNAs}

We classified bacterial CAU-anticodon-templating tRNA genes as templating methionine elongators, lysidinylated 
isoleucine elongators or initiators using TFAM version $1.4[10,11]$ with a general bacterial model for this purpose based on a previously published analysis [12].

\section{Structural annotation of 3 '- ends}

To annotate Sprinzl coordinates to the 3 '-end of each tRNAdb-CE sequence record, we implemented a dynamic programming algorithm to optimize base-pairing of the annotated 3 '-end of the mature tRNA in each record against its own annotated $5^{\prime}$-end and trailer sequence.

For each sequence record we obtained the $5^{\prime}$-most seven bases of the annotated acceptor stem sequence and reversed it to obtain sequence $x$. Given sequence $x$, we computed its optimal pairing against a second sequence $y$ defined by the last 12 bases of the annotated 3 '-end and the first five bases of the annotated 3 '-trailer using the simple dynamic programming algorithm described here.

Let $x$ and $y$ be finite sequences over the alphabet $\Sigma=\{A, C, G, U\}$, with lengths $m$ and $n$ respectively. We compute a matrix $H$ whose elements are specified as follows:

$$
\begin{aligned}
& H(i, 1)=0 \quad \forall i \in[1, m] \\
& H(1, j)=0 \quad \forall j \in[1, n] ; \\
& H(i, j)=\max (D, U, L) \forall i \in[2, m], \forall j \in[2, n] \\
& D=H(i-1, j-1)+s\left(x_{i}, y_{j}\right)+a\left(x_{i}, y_{j}\right) ; \\
& U=H(i-1, j)+g \\
& L=H(i, j-1)+g .
\end{aligned}
$$

where $i$ and $j$ are integers, $x_{i}$ is the $i$ th base in sequence $x$ of length $m=7, y_{j}$ is the $j$ th base in sequence $y$ of length $n=17, s\left(x_{i}, y_{j}\right)=4$, for $\left(x_{i}, y_{j}\right) \in\{(A, U),(U, A)$, $(C, G),(G, C),(G, U),(U, G)\}$ and $s\left(x_{i}, y_{j}\right)=1$ otherwise, $a\left(x_{i}\right.$ $\left.y_{j}\right)$ is an annotation bonus if $x_{i}$ and $y_{j}$ were annotated as paired in tRNAdb-CE, $g=-5$ is a linear gap penalty, and $H(i, j)$ is the maximum base-pairing score obtained on sequence prefixes $x[1, i]$ and $y[1, j]$. We compared results both with and without an annotation bonus, i.e. we recomputed $H(m, n)$ for every record using the bonus $a\left(x_{i}\right.$ $\left.y_{j}\right)=1$ or no bonus $a\left(x_{i} y_{j}\right)=0$.

\section{Statistics and visualization of genome size and CCA- templating data}

After reannotation, we considered a tRNA gene to template CCA if Sprinzl bases 74 through 76 contained the sequence CCA. We used genome size data downloaded as a "genome report" from NCBI Genome on October 26, 2015 [19] and visualized data using the Interactive Tree of Life $[50,51]$.

\section{Statistics of features in CAU-anticodon tRNAs}

We structurally aligned tRNA data using COVEA [52] with the TRNA2-prok.cm model from tRNAscan-SE v.1.23 [18] and analyzed feature statistics using FAST [53]. Complete commands to reproduce data in Tables 1 , 2, 3 and 4 are provided in the Supplement.

\section{Additional files}

Additional file 1: Reannotated tRNAdb-CE v.8 data in FastA format including TFAM scores and CCA reannotations. (TXT 43 MB)

Additional file 2: Structurally aligned data for subset of tRNAs with CAU anticodons, also containing TFAM scores with Silva model in "aligned FastA" format. (FAS $3 \mathrm{MB}$ )

Additional file 3: Expanded description of each additional file with usage. (TXT $3 \mathrm{~kb}$ )

Additional file 4: Prokaryotic genome size data from NCBI, downloaded on October 26, 2015. (TXT 10 MB)

Additional file 5: $\mathrm{NCBI}$ taxonomy data required by compute_heatmap.pl (GZ 14 MB)

Additional file 6: Installation code-base for the TFAM program. (ZIP 819 kb) Additional file 7: TFAM model used to reannotate tRNA functions in COVE format. To use this model file with TFAM, give its name as an option argument to the "-m" option. (TXT 1 MB)

Additional file 8: Perl script for structural reannotation of 3'ends. (TXT $5 \mathrm{~kb}$ )

Additional file 9: Perl script to generate full data browser and iTol input. (TXT $18 \mathrm{~kb}$ )

Additional file 10: Color definitions for clades to generate Fig. 1. (TXT 625 bytes)

Additional file 11: Phylogenetic tree in New Hampshire format of NCBI taxon IDs from NCBI Taxonomy corresponding to addresses_v4.txt.gz (TXT $8 \mathrm{~kb}$ )

Additional file 12: Web-browser based navigator of full dataset of results on frequencies of CCA-templating by tRNA functional class, organized by clades in NCBI taxonomy. (ZIP $548 \mathrm{~kb}$ )

Additional file 13: Static and searchable PDF with full dataset of results on frequencies of CCA-templating by tRNA functional class, organized by clades in NCBI taxonomy. (PDF 1 MB)

Additional file 14: List of FAST commands to reproduce frequency data shown in Tables 1, 2, 3 and 4. (TXT 4 kb)

\section{Abbreviations}

Asn: Asparagine; AUA/G: Codon sequences in messenger RNA; CAU: The anticodon sequence of initiator tRNAs and certain elongator tRNAs decoding codons as Met or Ile; CCA: The polyribonucleotide sequence ordinarily at the 3-prime end of mature tRNAs; fMet: N-Formylmethionine; Gln: Glutamine;

lle: Isoleucine; klle: Bacterial isoleucine tRNA elongators with CAU anticodons in which wobble position C34 is modified to lysidine; Met: Methionine; NCBI: National Center for Biotechnology Information; PDF: Portable Document Format; Pyl: Pyrrolysine; Sec: Selenocysteine; TFAM: The "tRNA Family" tRNA function classifier; tRNA: transfer ribonucleic acid; tRNAdb-CE: tRNA database Curated by Experts; Tyr: Tyrosine

\section{Acknowledgements}

Not applicable.

\section{Funding}

We thank the support of NSF grant 1344279 to DHA and NIH grants, 1 R01 GM114343, 5U01 GM108972, and 1R01 GM068561 to YMH.

\section{Availability of data and material}

All data generated or analysed during this study and supporting the conclusions of this article are included within the article and its Additional 
files $1,2,3,4,5,6,7,8,9,10,11,12,13$ and 14, and have also been permanently archived in the UC Merced DASH collection of the Merritt digital library service provided by the UC Curation Center with permanent identifier doi://10.6071/m3wc7k and URL http://n2t.net/ark:/b6071/m3wc7k.

\section{Authors' contributions}

DHA and YMH made substantial contributions to conception and design of the study. DHA acquired and analyzed data. DHA and YMH interpreted data. DHA and YMH drafted the manuscript and revised it critically for important intellectual content. DHA and YMH gave final approval of the version to be published, take public responsibility for appropriate portions of the content and agree to be accountable for all aspects of the work in ensuring that questions related to the accuracy or integrity of any part of the work are appropriately investigated and resolved.

\section{Competing interests}

The authors declare that they have no competing interests

\section{Ethics approval and consent to participate}

Not applicable.

\section{Consent for publication}

Not applicable.

\section{Author details}

${ }^{1}$ Program in Quantitative and Systems Biology, University of California, 5200 North Lake RoadCA 95343 Merced, USA. ${ }^{2}$ Molecular and Cell Biology Unit, School of Natural Sciences, University of California, 5200 North Lake Road, Merced, CA 95343, USA. ${ }^{3}$ Department of Biochemistry and Molecular Biology, Thomas Jefferson University, 233 South 10th Street, BLSB 220, Philadelphia, PA 19107, USA.

\section{Received: 8 July 2016 Accepted: 18 November 2016}

\section{Published online: 08 December 2016}

\section{References}

1. Betat $\mathrm{H}$, Morl M. The CCA-adding enzyme: A central scrutinizer in tRNA quality control. Bioessays. 2015;37(9):975-82.

2. Betat $\mathrm{H}$, Rammelt $\mathrm{C}$, Morl M. tRNA nucleotidyltransferases: ancient catalysts with an unusual mechanism of polymerization. Cell Mol Life Sci. 2010;67(9): 1447-63.

3. Vortler S, Morl M. tRNA-nucleotidyltransferases: highly unusual RNA polymerases with vital functions. FEBS Lett. 2010;584(2):297-302.

4. Sprinzl M, Horn C, Brown M, loudovitch A, Steinberg S. Compilation of tRNA sequences and sequences of tRNA genes. Nucleic Acids Res. 1998;26(1):148-53.

5. Suzuki T, Miyauchi K. Discovery and characterization of tRNAlle lysidine synthetase (TilS). FEBS Lett. 2010;584(2):272-7.

6. Fabret C, Dervyn E, Dalmais B, Guillot A, Marck C, Grosjean H, Noirot P. Life without the essential bacterial tRNA lle2-lysidine synthetase Tils: a case of tRNA gene recruitment in Bacillus subtilis. Mol Microbiol. 2011;80(4):1062-74.

7. Muramatsu T, Nishikawa K, Nemoto F, Kuchino Y, Nishimura S, Miyazawa T, Yokoyama S. Codon and amino-acid specificities of a transfer RNA are both converted by a single post-transcriptional modification. Nature. 1988; 336(6195):179-81.

8. Mandal D, Kohrer C, Su D, Russell SP, Krivos K, Castleberry CM, Blum P, Limbach PA, Soll D, RajBhandary UL. Agmatidine, a modified cytidine in the anticodon of archaeal tRNA(lle), base pairs with adenosine but not with guanosine. Proc Natl Acad Sci U S A. 2010;107(7):2872-7.

9. Ardell DH. Computational analysis of tRNA identity. FEBS Lett. 2010:584(2): 325-33.

10. Ardell DH, Andersson SGE. TFAM detects co-evolution of tRNA identity rules with lateral transfer of histidyl-tRNA synthetase. Nucleic Acids Res. 2006; 34(3):893-904

11. Tåquist $H$, Cui $Y$, Ardell DH. TFAM 1.0: an online tRNA function classifier. Nucleic Acids Res. 2007;35(Web Server issue):W350-3.

12. Silva FJ, Belda E, Talens SE. Differential annotation of tRNA genes with anticodon CAT in bacterial genomes. Nucleic Acids Res. 2006;34(20): 6015-22.

13. Freyhult E, Cui Y, Nilsson O, Ardell DH. New computational methods reveal tRNA identity element divergence between Proteobacteria and Cyanobacteria. Biochimie. 2007;89(10):1276-88.
14. Amrine $\mathrm{KCH}$, Swingley WD, Ardell DH. tRNA signatures reveal a polyphyletic origin of SAR11 strains among alphaproteobacteria. PLoS Comput Biol. 2014;10(2):e1003454

15. Abe T, Ikemura T, Ohara Y, Uehara H, Kinouchi M, Kanaya S, Yamada Y, Muto A, Inokuchi $H$. tRNADB-CE: tRNA gene database curated manually by experts. Nucleic Acids Res. 2009:37(Database issue):D163-8.

16. Abe T, Inokuchi H, Yamada Y, Muto A, Iwasaki Y, Ikemura T. tRNADB-CE: tRNA gene database well-timed in the era of big sequence data. Front Genet. 2014:5:114.

17. Laslett D, Canback B. ARAGORN, a program to detect tRNA genes and tmRNA genes in nucleotide sequences. Nucleic Acids Res. 2004;32(1):11-6.

18. Lowe TM, Eddy SR. tRNAscan-SE: a program for improved detection of transfer RNA genes in genomic sequence. Nucleic Acids Res. 1997;25(5):955-64.

19. NCBI Resource Coordinators. Database resources of the National Center for Biotechnology Information. Nucleic Acids Res. 2014;42(Database issue):D7-D17.

20. Hansen AK, Moran NA. Altered tRNA characteristics and 3' maturation in bacterial symbionts with reduced genomes. Nucleic Acids Res. 2012;40(16): 7870-84.

21. Marck C, Grosjean H. tRNomics: analysis of tRNA genes from 50 genomes of Eukarya, Archaea, and Bacteria reveals anticodon-sparing strategies and domain-specific features. RNA. 2002;8(10):1189-232.

22. Lee CP, RajBhandary UL. Mutants of Escherichia coli initiator tRNA that suppress amber codons in Saccharomyces cerevisiae and are aminoacylated with tyrosine by yeast extracts. Proc Natl Acad Sci U S A. 1991;88(24):11378-82.

23. Meinnel T, Blanquet S. Maturation of pre-tRNA(fMet) by Escherichia coli RNase $\mathrm{P}$ is specified by a guanosine of the 5 '-flanking sequence. J Biol Chem. 1995:270(26):15908-14.

24. Wegscheid B, Hartmann RK. The precursor tRNA 3'-CCA interaction with Escherichia coli RNase P RNA is essential for catalysis by RNase P in vivo. RNA. 2006;12(12):2135-48.

25. Wegscheid $B$, Hartmann RK. In vivo and in vitro investigation of bacteria type B RNase P interaction with tRNA 3'-CCA. Nucleic Acids Res. 2007;35(6): 2060-73.

26. Reiter NJ, Osterman A, Torres-Larios A, Swinger KK, Pan T, Mondragon A. Structure of a bacterial ribonuclease $\mathrm{P}$ holoenzyme in complex with tRNA. Nature. 2010;468(7325):784-9.

27. Shi PY, Weiner AM, Maizels N. A top-half tDNA minihelix is a good substrate for the eubacterial CCA-adding enzyme. RNA. 1998;4(3):276-84.

28. Ehrenberg $M$, Kurland CG. Costs of accuracy determined by a maximal growth rate constraint. Q Rev Biophys. 1984;17(1):45-82.

29. Klumpp S, Scott M, Pedersen S, Hwa T. Molecular crowding limits translation and cell growth. Proc Natl Acad Sci U S A. 2013;110(42):16754-9.

30. Vind J, Sorensen MA, Rasmussen MD, Pedersen S. Synthesis of proteins in Escherichia coli is limited by the concentration of free ribosomes. Expression from reporter genes does not always reflect functional mRNA levels. J Mol Biol. 1993;231(3):678-88.

31. Hersch SJ, Elgamal S, Katz A, Ibba M, Navarre WW. Translation initiation rate determines the impact of ribosome stalling on bacterial protein synthesis. J Biol Chem. 2014;289(41):28160-71.

32. Pop C, Rouskin S, Ingolia NT, Han L, Phizicky EM, Weissman JS, Koller D. Causal signals between codon bias, mRNA structure, and the efficiency of translation and elongation. Mol Syst Biol. 2014:10:770.

33. Dong HJ, Nilsson L, Kurland CG. Co-variation of tRNA abundance and codon usage in Escherichia coli at different growth rates. J Mol Biol. 1996;260(5):649-63.

34. Aiyar SE, Gaal T, Gourse RL. rRNA promoter activity in the fast-growing bacterium Vibrio natriegens. J Bacteriol. 2002;184(5):1349-58.

35. Dupasquier M, Kim S, Halkidis K, Gamper H, Hou YM. tRNA integrity is a prerequisite for rapid CCA addition: implication for quality control. J Mo Biol. 2008;379(3):579-88.

36. Hou YM. CCA addition to tRNA: implications for tRNA quality control. IUBMB Life. 2010;62(4):251-60

37. Elf J, Nilsson D, Tenson T, Ehrenberg M. Selective charging of tRNA isoacceptors explains patterns of codon usage. Science. 2003;300(5626): $1718-22$.

38. O'Farrell PH. The suppression of defective translation by ppGpp and its role in the stringent response. Cell. 1978:14(3):545-57.

39. Gamper HB, Masuda I, Frenkel-Morgenstern M, Hou YM. Maintenance of protein synthesis reading frame by EF-P and m(1)G37-tRNA. Nat Commun. 2015;6:7226. 
40. Gallant JA, Lindsley D. Ribosomes can slide over and beyond "hungry" codons, resuming protein chain elongation many nucleotides downstream. Proc Natl Acad Sci U S A. 1998;95(23):13771-6.

41. Drummond DA, Wilke CO. The evolutionary consequences of erroneous protein synthesis. Nat Rev Genet. 2009;10(10):715-24.

42. Burnap RL. Systems and Photosystems: Cellular Limits of Autotrophic Productivity in Cyanobacteria. Front Bioeng Biotechnol. 2015;3:1.

43. Esquivel MG, Pinto TS, Marin-Navarro J, Moreno J. Substitution of tyrosine residues at the aromatic cluster around the betaA-betaB loop of rubisco small subunit affects the structural stability of the enzyme and the in vivo degradation under stress conditions. Biochemistry. 2006:45(18):5745-53.

44. Wegener KM, Singh AK, Jacobs JM, Elvitigala T, Welsh EA, Keren N, Gritsenko MA, Ghosh BK, Camp DG, Smith RD, et al. Global proteomics reveal an atypical strategy for carbon/nitrogen assimilation by a cyanobacterium under diverse environmental perturbations. Mol Cell Proteomics. 2010; 9(12):2678-89

45. Wydau S, van der Rest G, Aubard C, Plateau P, Blanquet S. Widespread Distribution of Cell Defense against d-Aminoacyl-tRNAs. J Biol Chem. 2009: 284(21):14096-104

46. Bailly M, Blaise M, Lorber B, Becker HD, Kern D. The transamidosome: a dynamic ribonucleoprotein particle dedicated to prokaryotic tRNA-dependent asparagine biosynthesis. Mol Cell. 2007:28(2):228-39.

47. Becker HD, Kern D. Thermus thermophilus: a link in evolution of the tRNA-dependent amino acid amidation pathways. Proc Natl Acad Sci U S A. 1998;95(22):12832-7.

48. Curnow AW, Ibba M, Soll D. tRNA-dependent asparagine formation. Nature. 1996;382(6592):589-90.

49. Gagnon Y, Lacoste L, Champagne N, Lapointe J. Widespread use of the glu-tRNAGIn transamidation pathway among bacteria. A member of the alpha purple bacteria lacks glutaminyl-trna synthetase. J Biol Chem. 1996;271(25):14856-63.

50. Letunic I, Bork P. Interactive Tree Of Life (iTOL): an online tool for phylogenetic tree display and annotation. Bioinformatics. 2007;23(1):127-8.

51. Letunic I, Bork P. Interactive Tree Of Life v2: online annotation and display of phylogenetic trees made easy. Nucleic Acids Res. 2011;39(Web Server issue):W475-8.

52. Eddy SR, Durbin R. RNA sequence analysis using covariance models. Nucleic Acids Res. 1994;22(11):2079-88.

53. Lawrence TJ, Kauffman KT, Amrine KCH, Carper DL, Lee RS, Becich PJ, Canales CJ, Ardell DH. FAST: FAST Analysis of Sequences Toolbox. Front Genet. 2015;6:172

\section{Submit your next manuscript to BioMed Central and we will help you at every step:}

- We accept pre-submission inquiries

- Our selector tool helps you to find the most relevant journal

- We provide round the clock customer support

- Convenient online submission

- Thorough peer review

- Inclusion in PubMed and all major indexing services

- Maximum visibility for your research

Submit your manuscript at www.biomedcentral.com/submit

) Biomed Central 